\title{
Perfil populacional de pacientes com disartria atendidos em hospital terciário
}

\author{
Populational profile of dysarthric patients assisted in a tertiary \\ hospital
}

Ariella Fornachari Ribeiro', Karin Zazo Ortiz ${ }^{2}$

\begin{abstract}
RESUMO
Objetivo: Caracterizar o perfil populacional dos pacientes disártricos atendidos em um hospital terciário e apresentar as alterações mais frequentes nas cinco bases motoras que estão comumente alteradas nas disartrias. Métodos: Este estudo foi realizado por meio da análise da aplicação de um protocolo de disartria, composto por tarefas que avaliam a respiração, a fonação, a ressonância, a articulação e a prosódia. Foram escolhidos aleatoriamente 60 protocolos de disartria, que foram aplicados aos pacientes submetidos à avaliação, no ambulatório de Distúrbios da Comunicação do Hospital São Paulo - Hospital Escola da Universidade Federal de São Paulo - Escola Paulista de Medicina. A análise dos dados foi realizada de maneira descritiva. Resultados: Em relação ao perfil populacional dos pacientes disártricos atendidos em um hospital terciário, observamos prevalência do sexo masculino, acometidos principalmente entre 20 e 50 anos de idade. Os tipos mais comuns de disartria foram a flácida e a disartria do neurônio motor superior unilateral. A etiologia mais prevalente para este distúrbio de fala foi o AVC. Em relação às bases motoras avaliadas, houve predomínio de respiração do tipo mista, a rouquidão foi a característica vocal mais encontrada; o movimento velar esteve predominantemente adequado, apesar da identificação de hipernasalidade discreta. A articulação mostrou-se bastante comprometida nos quadros avaliados e houve predomínio de alteração discreta da prosódia na população investigada. Conclusão: Foi possível estabelecer o perfil de pacientes disártricos atendidos em Hospital terciário em nosso meio, que apresentaram predominantemente as seguintes características: ciclos respiratórios curtos, voz rouca, hipernasal, alteração moderada a grave de articulação, discreta alteração de prosódia e velocidade lenta de fala.
\end{abstract}

Descritores: Disartria; Distúrbios da voz; Acústica da fala; Fala; Qualidade da voz

\section{INTRODUÇÃO}

A disartria é um distúrbio de fala, resultante de alterações no controle muscular dos mecanismos envolvidos em sua produção, originado por uma lesão do Sistema Nervoso Central ou Periférico que acarreta em alterações na emissão oral, devido a uma paralisia, fraqueza ou falta de coordenação dos músculos da fala ${ }^{(1)}$.

Uma disartria pode ser secundária a uma tumoração do cérebro, cerebelo ou tronco encefálico, a doenças infecciosas,

Trabalho realizado no Núcleo de Investigação Fonoaudiológica em Neuropsicolinguística do Departamento de Fonoaudiologia da Universidade Federal de São Paulo - UNIFESP - São Paulo (SP), Brasil.

(1) Pós-graduanda (Mestrado) do Programa de Pós-Graduação em Ciências da Universidade de São Paulo - USP - São Paulo (SP), Brasil.

(2) Doutora, Professora Adjunto da Disciplina de Distúrbios da Comunicação Humana do Departamento de Fonoaudiologia da Universidade Federal de São Paulo - UNIFESP - São Paulo (SP), Brasil.

Endereço para correspondência: Karin Zazo Ortiz. R. Cunha, 111, conj. 73, Vila Clementino, São Paulo (SP), Brasil, CEP: 04037-030. E-mail: karinortiz.fono@epm.br

Recebido em: 24/11/2008; Aceito em: 25/5/2009 metabólicas, tóxicas ou degenerativas do sistema nervoso ou do sistema muscular. Geralmente, é uma sequela comum de lesões cerebrais não progressivas, consequentes de uma lesão vascular cerebral ou de uma traumatismo cranioencefálico, bem como de doenças progressivas neuromusculares ou que atingem o Sistema Extrapiramidal ${ }^{(2-5)}$.

Existem vários tipos de disartria, de acordo com o local da lesão: disartria flácida, disartria espástica, disartria do neurônio motor superior unilateral, disartria hipocinética, disartria hipercinética, disartria atáxica e disartria mista ${ }^{(4)}$. Cada um desses tipos apresenta características peculiares que envolvem o desempenho anormal das estruturas pulmonares, laríngeas, faríngeas e da cavidade oral que agrupadas correspondem às bases fonoarticulatórias, responsáveis pela produção de uma fala inteligível. Alterações nessas bases são suficientes para causar uma alteração na inteligibilidade de fala ininteligível do mesmo ${ }^{(6-7)}$.

Sabe-se que o processo fonoarticulatório em pacientes com disartria tende a ser prejudicado e, apesar da variação dos quadros, é comum encontrar em quase todos os pacientes disártricos: diminuição da velocidade da fala, imprecisão 
articulatória, fala lenta e irregular, monoaltura e monointensidade $^{(8-9)}$.

Em nosso meio, o número de publicações sobre o assunto ainda é reduzido e não foram encontrados artigos que apresentassem o perfil populacional de pacientes disártricos, especialmente em relação às cinco bases motoras da fala.

Inúmeras são as doenças neurológicas que podem causar a disartria $^{(9)}$. No entanto, considerando-se a gama de possibilidades, algumas doenças são mais comuns do que outras, podendo desta forma, causar disartrias que sejam mais prevalentes em uma determinada população. Assim, seria importante verificarmos se há quadros mais comuns ou manifestações mais evidentes em um determinado grupo.

Dessa forma, esta pesquisa teve como objetivo caracterizar o perfil populacional dos pacientes disártricos atendidos em um hospital terciário e apresentar as alterações mais freqüentes nas cinco bases motoras que estão comumente alteradas nas disartrias.

\section{MÉTODOS}

\section{Instrumento}

Este estudo foi realizado por meio da análise da aplicação de um protocolo de disartria, previamente publicado, composto por tarefas que avaliam a respiração, a fonação, a ressonância, a articulação e a prosódia, ou seja, as cinco grandes bases que promovem uma fala inteligível ${ }^{(4)}$. As variáveis investigadas em cada uma das bases avaliadas são descritas a seguir.

- Respiração: tipo (abdominal, mista, torácica, clavicular e/ou inversa) e velocidade da respiração. Tempos máximos de fonação do /a/, do /i/, do /s/ e do /z/ e a relação s/z. Na avaliação de palavras por expiração, realizamos dois tipos de procedimentos: média do número de números emitidos por respiração na contagem de 01 a 20 , e média de número de palavras emitidas por expiração, na leitura de um texto padrão.

- Fonação: análise perceptual auditiva da qualidade vocal; análise do ataque vocal; da loudness e do pitch.

- Ressonância: movimento velar e nasalidade. As alterações de ressonância, quando presentes, foram ainda classificadas como discreta, leve, moderada ou grave.

- Articulação: foram analisados os movimentos labiais, linguais, mandibulares isolados e em atividades de fala. Também se analisou a inteligibilidade de fala na emissão de monossílabos e de sentenças.

- Prosódia: manutenção da sílaba tônica, da entonação da sentença e entre as sentenças. Na investigação desta base motora analisou-se, também, a velocidade de fala.

\section{Casuística}

Foram selecionados 60 protocolos de disartria que haviam sido aplicados aos pacientes submetidos à avaliação, no ambulatório de Distúrbios Neurológicos Adquiridos da Fala e da Linguagem do Hospital São Paulo (HSP) - Hospital Escola da Universidade Federal de São Paulo, um dos serviços de referência para o atendimento de distúrbios da comunicação em pacientes com lesão cerebral adquirida na cidade de São Paulo.
Os pacientes expostos à avaliação formal, segundo o protocolo de diagnóstico de disartria, são pacientes que procuraram o serviço de atendimento fonoaudiológico voluntariamente no período de 2002 a 2008. Neste período, foram avaliados 104 pacientes por meio deste protocolo. Foram escolhidos para este estudo apenas os protocolos de pacientes que não se encontravam mais em acompanhamento no ambulatório do HSP e, no momento da coleta dos dados, compunham os grupos de altas fonoaudiológicas, óbitos e desistências, sendo este um estudo retrospectivo. Além disso, protocolos que tivessem mais de $20 \%$ dos dados faltantes foram excluídos do estudo. Desta forma, foram analisados os protocolos de 60 pacientes, ou seja, $57 \%$ dos casos avaliados. Os protocolos dos pacientes que se encontravam em acompanhamento foram excluídos, a fim de obter-se maior fidedignidade na tabulação dos dados.

\section{Procedimentos}

Este estudo foi aprovado pelo Comitê de Ética em Pesquisa da Universidade Federal de São Paulo/Hospital São Paulo sob protocolo número 1525/04.

Inicialmente, os indivíduos pertencentes à amostra foram descritos, um a um, de forma que se identificasse o sexo e idade de cada paciente, o diagnóstico fonoaudiológico quanto ao tipo de disartria e o tipo ou local da lesão que acarretou o quadro disártrico do paciente.

Foram analisados e tabulados os testes de cada uma das cinco bases motoras, estabelecendo assim o perfil da amostra em estudo. As bases foram analisadas na seguinte ordem: respiração, fonação, ressonância, articulação e, por último, analisou-se a prosódia.

A análise descritiva dos dados coletados foi realizada após a tabulação de todos os dados de interesse deste estudo, tendo os mesmos sido submetidos a tratamento estatístico. Todos os gráficos foram construídos segundo o pacote estatístico SPSS (Statistical Pakage for the Social Science) 11.5.1 para Windows.

\section{RESULTADOS}

\section{Caracterização da amostra}

A análise do perfil populacional demonstrou que, na amostra, composta por 60 protocolos, foram encontrados 17 protocolos aplicados a mulheres e 43 aplicados a homens. A média de idade desses pacientes foi de 52 anos.

Desses 60 protocolos avaliados, 36 apresentaram o diagnóstico de disartria, com seu subtipo especificado e a etiologia mais encontrada para esta alteração de fala, tanto em homens quanto em mulheres foi a lesão vascular encefálica.

Nas Tabelas 1, 2 e 3 encontra-se a idade dos pacientes avaliados segundo o sexo, o tipo de Disartria e a descrição da lesão e/ou etiologia neurológica.

\section{Resultados encontrados em cada uma das bases motoras avaliadas}

Respiração

Em relação às provas que avaliam a respiração (Tabela 
Tabela 1. Idade dos pacientes avaliados segundo a variável sexo

\begin{tabular}{|c|c|c|c|c|c|c|}
\hline \multirow[t]{2}{*}{ Idade dos pacientes (anos) } & \multicolumn{2}{|c|}{ Sexo masculino } & \multicolumn{2}{|c|}{ Sexo feminino } & \multicolumn{2}{|c|}{ Total } \\
\hline & $\mathrm{N}$ & $\%$ & $\mathrm{~N}$ & $\%$ & $\mathrm{~N}$ & $\%$ \\
\hline Menos que 20 & 2 & 4,6 & 1 & 5,8 & 3 & 5 \\
\hline $20-50$ & 15 & 34,8 & 7 & 41,1 & 22 & 36,6 \\
\hline $51-65$ & 12 & 27,9 & 7 & 41,1 & 19 & 31,6 \\
\hline Mais que 65 & 14 & 32,5 & 2 & 11,7 & 16 & 26,6 \\
\hline Total & 43 & 100 & 17 & 100 & 60 & 100 \\
\hline
\end{tabular}

Tabela 2. Descrição de pacientes segundo o tipo de disartria

\begin{tabular}{|c|c|c|c|c|c|c|}
\hline \multirow[t]{2}{*}{ Tipo de disartria } & \multicolumn{2}{|c|}{ Sexo masculino } & \multicolumn{2}{|c|}{ Sexo feminino } & \multicolumn{2}{|c|}{ Total } \\
\hline & $\mathrm{N}$ & $\%$ & $\mathrm{~N}$ & $\%$ & $\mathrm{~N}$ & $\%$ \\
\hline Flácida & 12 & 27,9 & 8 & 47 & 20 & 33,3 \\
\hline Espástica & 6 & 13,9 & 4 & 23,5 & 10 & 16,6 \\
\hline Hipercinética & 2 & 4,6 & 0 & 0 & 2 & 3,3 \\
\hline Hipocinética & 4 & 9,3 & 2 & 11,7 & 6 & 10 \\
\hline Neurônio motor superior unilateral & 12 & 27,9 & 2 & 11,7 & 14 & 23,3 \\
\hline Mista & 6 & 13,9 & 0 & 0 & 6 & 10 \\
\hline Não informada & 1 & 2,3 & 1 & 5,8 & 2 & 3,3 \\
\hline Total & 43 & 100 & 17 & 100 & 60 & 100 \\
\hline
\end{tabular}

Tabela 3. Descrição de pacientes segundo o tipo de lesão/etiologia

\begin{tabular}{|c|c|c|c|c|c|c|}
\hline \multirow[t]{2}{*}{ Etiologia } & \multicolumn{2}{|c|}{ Sexo masculino } & \multicolumn{2}{|c|}{ Sexo feminino } & \multicolumn{2}{|c|}{ Total } \\
\hline & $\mathrm{N}$ & $\%$ & $\mathrm{~N}$ & $\%$ & $\mathrm{~N}$ & $\%$ \\
\hline AVC & 29 & 67,4 & 9 & 53 & 38 & 63,3 \\
\hline TCE & 5 & 11,6 & 0 & 0 & 5 & 8,3 \\
\hline Doenças progressivas & 7 & 16,2 & 7 & 16,2 & 14 & 23,3 \\
\hline Em investigação neurológica & 2 & 4,6 & 1 & 5,8 & 3 & 5 \\
\hline Total & 43 & 100 & 17 & 100 & 60 & 100 \\
\hline
\end{tabular}

Legenda: $\mathrm{AVC}$ = acidente vascular cerebral; TCE = traumatismo cranioencefálico

4), verificou-se que a maioria dos pacientes avaliados nos protocolos possuía respiração mista $(48,3 \%)$.

A média da velocidade da respiração desses pacientes foi de 20,18 ciclos por minuto, sendo que não houve diferenças nas médias encontradas entre os sexos: masculino e feminino.

Verificou-se que nos tempos máximos fonatórios, as médias encontradas foram de dez segundos para o fonema /a/, dez segundos para o fonema /i/, 8,5 segundos para o fonema /s/ e 8 segundos para o fonema /z/. Além disso, verificou-se que as médias dos tempos máximos fonatórios das mulheres foi inferior aos tempos máximos emitidos pelos homens.

A média da relação s/z dos pacientes foi de 1,3, demonstrando que os mesmos apresentam importante incoordenação pneumofonoarticulatória. Os pacientes apresentaram uma média de 7,7 palavras por expiração na contagem de números e 6,8 palavras por expiração na elaboração de discurso.

Tabela 4. Tipo de respiração dos pacientes

\begin{tabular}{|c|c|c|c|c|c|c|}
\hline \multirow[t]{2}{*}{ Tipo de respiração } & \multicolumn{2}{|c|}{ Sexo masculino } & \multicolumn{2}{|c|}{ Sexo feminino } & \multicolumn{2}{|c|}{ Total } \\
\hline & $\mathrm{N}$ & $\%$ & $\mathrm{~N}$ & $\%$ & $\mathrm{~N}$ & $\%$ \\
\hline Abdominal & 13 & $30,2 \%$ & 4 & $23,5 \%$ & 17 & $28,8 \%$ \\
\hline Clavicular & 1 & $2,3 \%$ & 0 & $0 \%$ & 1 & $1,6 \%$ \\
\hline Torácica & 9 & $21 \%$ & 3 & $17,6 \%$ & 12 & $20 \%$ \\
\hline Mista & 19 & $44 \%$ & 10 & $58,8 \%$ & 29 & $48,3 \%$ \\
\hline Não informada & 1 & $2,3 \%$ & 0 & $0 \%$ & 1 & $1,6 \%$ \\
\hline Total & 43 & $100 \%$ & 17 & $100 \%$ & 60 & $100 \%$ \\
\hline
\end{tabular}




\section{Fonação}

As qualidades vocais dos pacientes estão representadas na Figura 1. Pôde-se perceber que, enquanto aproximadamente $70 \%$ do sexo masculino apresentaram qualidade vocal rouca, o sexo feminino apresentou predominantemente qualidade vocal hipernasal (70\%). Os outros aspectos envolvidos na fonação, em avaliação subjetiva, revelaram que o perfil fonatório dos indivíduos foi: ataque vocal isocrônico $(71,7 \%)$, intensidade vocal adequada $(53 \%)$, seguida de intensidade vocal baixa com $43 \%$ e predominância de pitch grave em $55 \%$ dos casos avaliados.

\section{Ressonância}

Na avaliação da ressonância, foi realizada a classificação do movimento velar e da nasalidade dos pacientes. A Figura 2 mostra os resultados obtidos nessas avaliações.

\section{Articulação}

A média de inteligibilidade de fala para monossílabos dos indivíduos foi de $57 \%$, enquanto a média de inteligibilidade para sentenças foi de $50,3 \%$.

Conforme se visualiza na Figura 3, nas tarefas que envolvem os movimentos articulatórios, os indivíduos apresentam

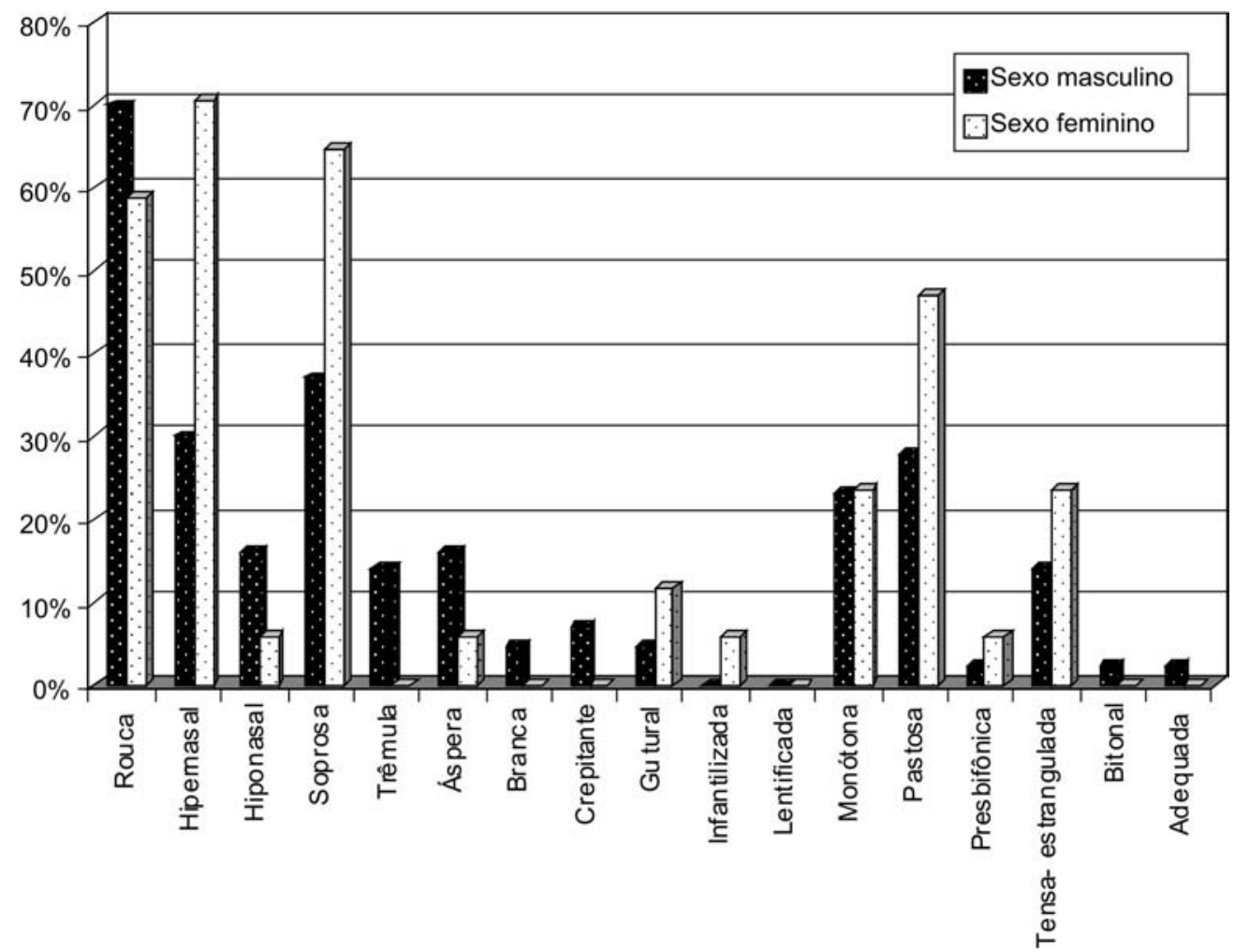

Figura 1. Qualidade Vocal dos pacientes avaliados distribuída conforme o sexo

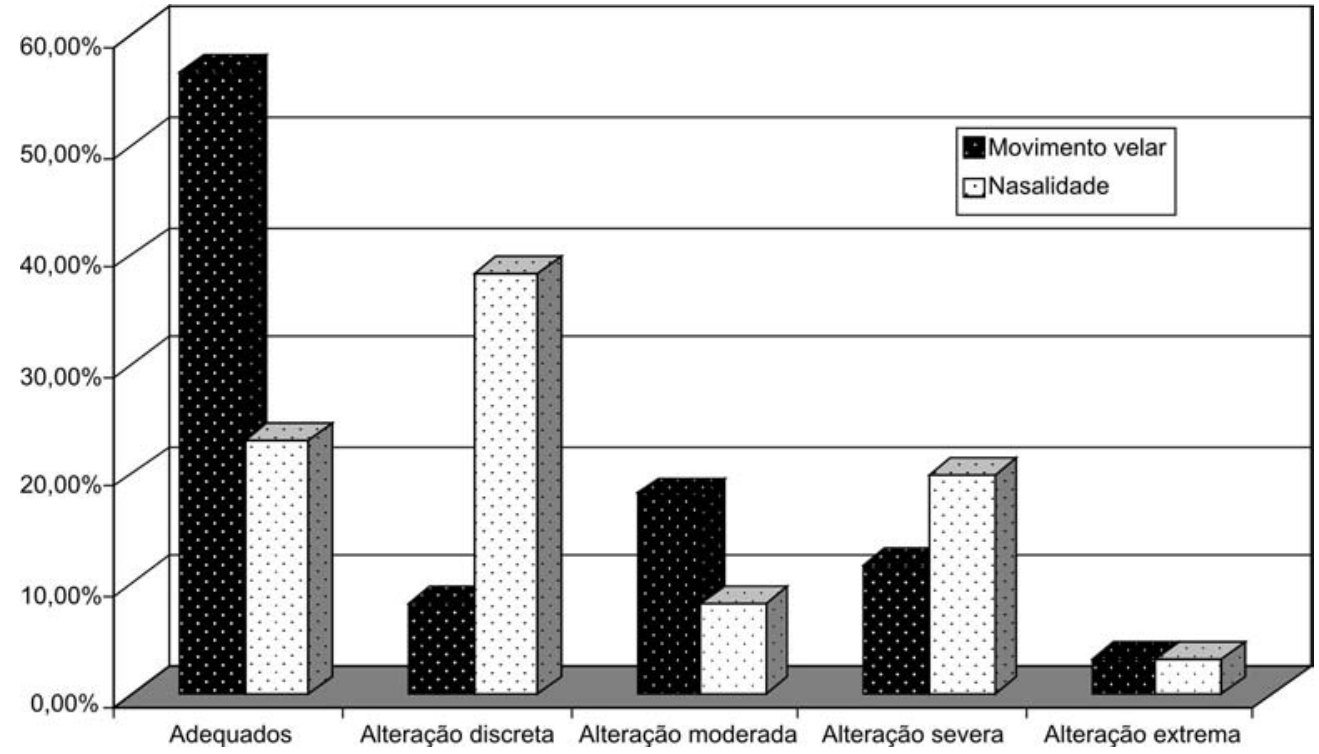

Figura 2. Resultados percentuais da avaliação do movimento velar e nasalidade dos indivíduos 


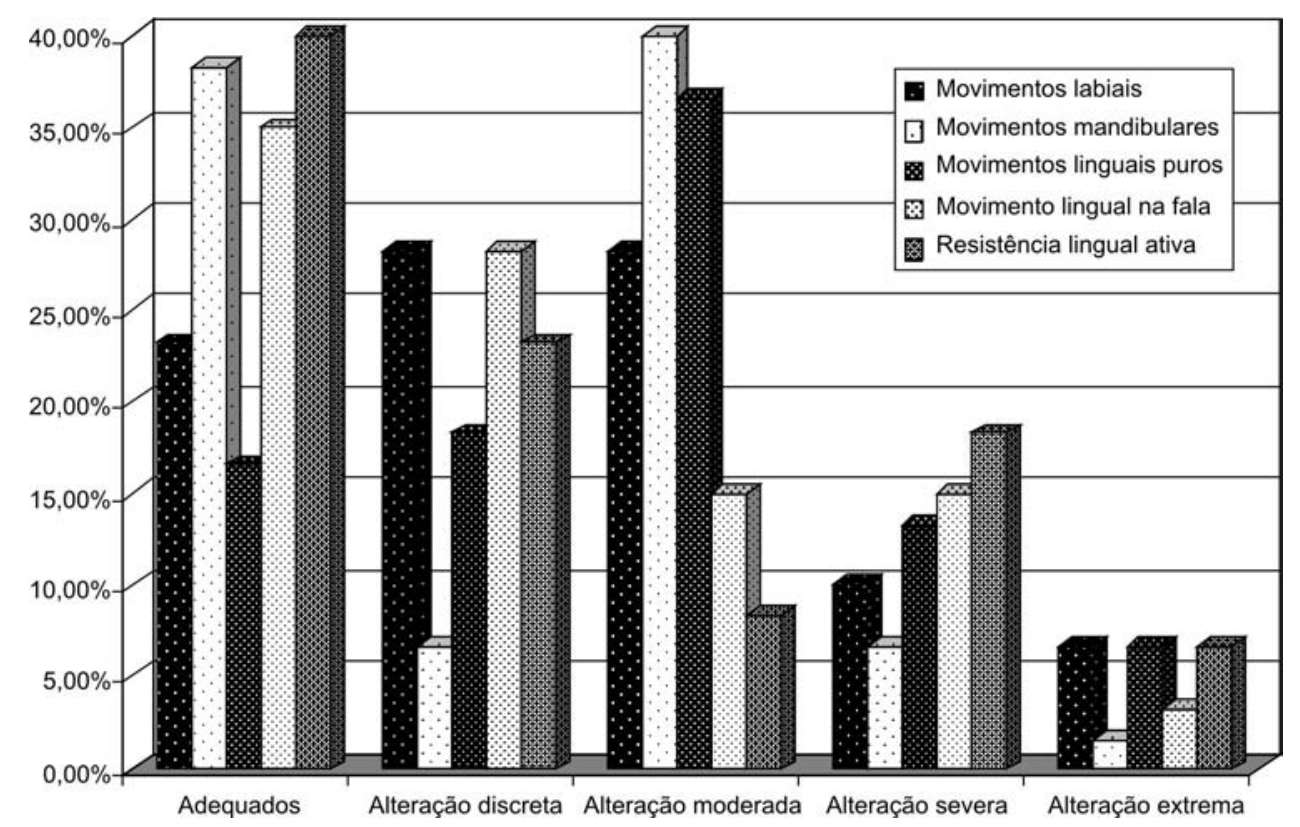

Figura 3. Resultados percentuais da avaliação dos indivíduos nas tarefas de articulação

Tabela 5. Entonação e uso de sílabas tônicas na fala dos pacientes

\begin{tabular}{|c|c|c|c|c|c|c|}
\hline \multirow[t]{2}{*}{ Resultado da avaliação } & \multicolumn{2}{|c|}{ Sexo masculino } & \multicolumn{2}{|c|}{ Sexo feminino } & \multicolumn{2}{|c|}{ Total } \\
\hline & $\mathrm{N}$ & $\%$ & $\mathrm{~N}$ & $\%$ & $\mathrm{~N}$ & $\%$ \\
\hline Alteração discreta & 12 & 28 & 4 & 23,5 & 16 & 26,6 \\
\hline Alteração moderada & 7 & 16,2 & 1 & 5,8 & 8 & 13,3 \\
\hline Alteração severa & 1 & 2,3 & 1 & 5,8 & 2 & 3,3 \\
\hline Alteração extrema & 9 & 21 & 4 & 23,5 & 13 & 21,6 \\
\hline Adequados & 6 & 14 & 2 & 11,7 & 8 & 13,3 \\
\hline Não informado & 8 & 18,6 & 5 & 29,4 & 13 & 21,6 \\
\hline Total & 43 & 100 & 17 & 100 & 60 & 100 \\
\hline
\end{tabular}

alteração discreta e moderada quando avaliados os movimentos labiais ( $28 \%$ para cada alteração); $40 \%$ de indivíduos apresentam alteração moderada na avaliação dos movimentos mandibulares, aproximadamente $37 \%$ de pacientes apresentam alteração moderada na investigação de movimentos linguais isolados, $35 \%$ de sujeitos apresentam movimentos linguais durante a fala adequados e $40 \%$ realizaram a tarefa de resistência lingual ativa adequadamente.

\section{Prosódia}

$\mathrm{Na}$ avaliação da prosódia, foram investigadas as tarefas de velocidade de leitura e verificada a entonação das frases e entre as frases, bem como o uso de sílabas tônicas na fala dos pacientes. Os pacientes avaliados apresentaram uma média de 90 palavras por minuto durante a conversação ou leitura de um texto.

A Tabela 5 retrata os resultados encontrados na avaliação da entonação dos pacientes.

\section{DISCUSSÃO}

O objetivo deste estudo foi estabelecer um perfil populacional dos pacientes disártricos atendidos em Hospital Terciário.
Para tanto, o estudo constou de resultados descritivos, que serão discutidos a seguir.

Estudos revelam que a doença cérebro vascular é causa mais frequente de morbidades e óbitos de pacientes, no $\mathrm{Mu}-$ nicípio de São Paulo ${ }^{(10)}$ e é a terceira causa mais freqüente de morte, nos Estados Unidos ${ }^{(11-12)}$. Conforme observado na Tabela 2, os dados desta pesquisa relatam que mais da metade dos pacientes avaliados sofreu um acidente vascular encefálico, corroborando, portanto, a literatura consultada.

Existem divergências quanto ao sexo da população acometida. Algumas pesquisas retratam que o sexo masculino apresenta maior índice de lesões ${ }^{(10,13)}$, enquanto outros estudos evidenciam uma incidência maior no sexo feminino ${ }^{(14)}$. Com relação à faixa etária, os estudos também são bastante diversificados. Embora, existam relatos de que o risco de uma lesão vascular cerebral aumente com a idade ${ }^{(13)}$, algumas pesquisas encontram um índice maior de AVC em populações com idade média $^{(13-14)}$, ao passo que outros pesquisadores descreveram alta incidência de lesões vasculares em indivíduos entre 75 e $85 \operatorname{anos}^{(14)}$. No presente estudo, encontrou-se uma população com predomínio do sexo masculino, na faixa etária de 20 a 50 anos (Tabela 1).

O predomínio de pacientes do sexo masculino pode ainda 
ser justificado pelo fato de que a segunda etiologia mais encontrada nas avaliações ter sido o traumatismo cranioencefálico, que é sabidamente o acometimento de maior incidência nesse $\operatorname{sexo}^{(14-15)}$.

Em relação ao tipo de disartria, observamos predomínio das disartrias flácida e do Neurônio Motor Superior Unilateral (Tabela 3). De fato, a disartria do Neurônio Motor Unilateral é comum em pacientes com lesão isquêmica unilateral e a Disartria Flácida é também bastante descrita em casos de TCE, etiologia prevalente em nossa casuística.

\section{Comentários sobre os achados relativos às Bases Moto- ras da Fala}

\section{Respiração}

Desvios em várias dimensões perceptivas de fala podem ser utilizados como indicadores da adequação ou inadequação do suporte respiratório para a fala de um determinado paciente ${ }^{(16)}$.

Em pacientes neurológicos, o comprometimento geralmente se encontra na amplitude do movimento e na regularidade dos ciclos respiratórios e as alterações do padrão respiratório abrangem o início da emissão após o início da fase expiratória, excesso de pausas inspiratórias e uso de ar de reserva, entre outros, o que pode se refletir em incoordenação pneumofonoarticulatória $^{(2)}$. Esses achados combinam com os resultados do presente estudo, já que nesta análise, observou-se que quase metade dos pacientes apresentou respiração mista, seguida de respiração abdominal e respiração torácica (Tabela 4). Além disso, muitos pacientes avaliados apresentaram incoordenação pneumofonoarticulatória, que foi evidenciada durante a contagem de números e a elaboração de um discurso.

O tempo máximo de fonação (TMF) é obtido por meio de teste rotineiramente aplicado, com intuito de avaliar a eficiência glótica ${ }^{(17)}$. Os valores normais esperados para homens são: 20 segundos e, para as mulheres, 15 segundos ${ }^{(18-20)}$. Esses valores podem ser um pouco mais altos: 25 a 35 s para falantes masculinos e 15 a $25 \mathrm{~s}$ para falantes femininos ${ }^{(18)}$. As vogais utilizadas são /a/, /i/ e /u/ e as consoantes /s/ e /z/(18). A amostra desse estudo apresentou média de 9 segundos de TMF, o que claramente evidencia um comprometimento de fonte glótica. Além disso, sabe-se que para a emissão da vogal /a/, valores de Tempo Máximo de Fonação menores que dez segundos em adultos devem ser sempre considerados patológicos ${ }^{(4)}$.

$\mathrm{O} / \mathrm{s} /$ é uma medida do controle expiratório e o som de /z/ acrescenta à tarefa o componente laríngeo. Normalmente, a fricativa surda /s/ e a fricativa sonora /z/ são sustentadas, por um falante normal, em tempos aproximadamente iguais ou sendo a sustentação do /z/ ligeiramente superior ${ }^{(16)}$. Disso resulta uma proporção (relação s/z) que, para ser considerada normal, deve variar de 0,8 a 1,2. Essa relação fornece dados muito interessantes sobre a dinâmica da fonação e tem sido considerada como medida bastante fidedigna de avaliação da eficiência glótica ${ }^{(16)}$. Valores da relação s/z acima de 1,3 revelam falta de coaptação glótica ${ }^{(16)}$. A média da relação s/z dos pacientes encontrou-se dentro dos achados da literatura.

Além disso, os TMF dependem de uma série de fatores como o esforço realizado pelo indivíduo, sexo, idade, estatura, estado emocional e condições neurovegetativas ${ }^{(18)}$. Esses dados somados às referências anteriormente citadas justificam o fato dos homens apresentaram TMF superior aos TMF da população feminina.

Em relação à média da velocidade da respiração desses pacientes, relatos indicam que o cérebro promove, em média, uma inspiração a cada cinco segundos, ou seja, 12 ciclos por minuto $^{(17)}$, enquanto indivíduos disártricos podem apresentar entre 12 a $20 \operatorname{ciclos}^{(4)}$. Esta pesquisa corrobora os dados obtidos com pacientes disártricos, uma vez que se encontrou uma média de 20,18 ciclos por minuto nos indivíduos avaliados.

\section{Fonação}

A fonação normal requer um controle motor extremamente preciso. Qualquer fraqueza, lentidão ou incoordenação da musculatura laríngea pode ser percebida por intermédio da voz ${ }^{(6,13)}$.

Os julgamentos perceptivos fonatórios devem fornecer descrições confiáveis e válidas referentes aos vários aspectos relacionados às vozes normais e alteradas e, quando possível, deve ser associada à análise acústica ${ }^{(6)}$.

A análise perceptiva das dimensões alteradas da fala foi realizada em estudos com pacientes com doenças neurológicas $^{(6,18)}$. Os desvios perceptivos de fala dos níveis como pitch mais agudo, variação reduzida de pitch e loudness, variação maior de ritmo e voz muito soprosa, foram aspectos observados quando os indivíduos foram comparados com um grupo controle. Os autores concluíram que a avaliação perceptual auditiva é capaz de diferenciar a fala disártrica da fala normal.

Outros pesquisadores ${ }^{(19-22)}$ citam como características básicas de um paciente disártrico o esforço e a tensão ao falar, além de rouquidão e tremor vocal, variações de intensidade e frequência e interrupções da fonação e descrevem distúrbios fonatórios associados a patologias neurológicas. E outras pesquisas $^{(2,6)}$ relatam que os tipos de vozes mais frequentemente associados às doenças neurológicas são: tensa - estrangulada e soprosa.

Os achados do presente estudo concordam com a literatura no que diz respeito à variação reduzida do loudness desses pacientes, mas a amostra apresentou predominantemente qualidade vocal rouca e hipernasal, seguida da qualidade soprosa, intensidade vocal adequada, com pitch predominantemente grave (Figura 1).

\section{Ressonância}

As alterações de ressonância são relatadas frequentemente nos pacientes disártricos e refletem os distúrbios fisiopatológicos conhecidos de lentidão, fraqueza, incoordenação e tônus anormal do mecanismo velofarígeo ${ }^{(23)}$.

Nessa tarefa, verificamos que quase todos os pacientes avaliados apresentaram alterações discretas de ressonância, com maior índice de hipernasalidade (Figura 2).

De fato, a hipernasalidade é freqüente em pacientes com disartria $^{(23-24)}$. Devemos ponderar, no entanto, que os pareceres perceptivos sobre a ressonância são particularmente problemáticos, e foi sugerido por vários pesquisadores que as percepções de nasalidade podem ser influenciadas por outros fatores além de disfunção velofarígea, tais como: resistência ao fluxo aéreo nasal, imprecisão articulatória e distúrbios de outros mecanismos de produção de fala que possam causar 
hipernasalidade ao serem mascarados como, por exemplo, anormalidades fonatórias e da velocidade da fala ${ }^{(25-26)}$.

Articulação

A articulação envolve a interação complexa entre várias estruturas, entre elas lábios, língua, mandíbula e palato mole.

Os julgamentos perceptivos mostram uma conclusão desta interação complexa, ou seja, os sons produzidos ${ }^{(1,6)}$.

Embora, as anormalidades fonatórias e prosódicas sejam frequentemente encontradas no discurso de pacientes disártricos, as anormalidades na articulação são as características predominantemente mais desviadas, afetando principalmente a produção das consoantes ${ }^{(1,4)}$.

Quando se avaliou este complexo sistema articulatório observou-se que os pacientes apresentaram alteração moderada nos movimentos labiais, mandibulares e linguais puros e, movimentos adequados na tarefa de resistência lingual e nos movimentos linguais durante a produção de fala (Figura 3).

Durante a emissão das sílabas /pa/, /ta/ e /ka/ (sílabas também utilizadas no protocolo desta pesquisa), indivíduos com disartria apresentam um padrão homogêneo de anormalidades dos movimentos articulatórios, incluindo prolongações das sílabas, redução da velocidade e amplitude dos movimentos ${ }^{(27)}$. Esses achados corroboram com o presente estudo.

Em relação à inteligibilidade de fala, constatou-se que os pacientes apresentaram uma média de $27 \%$ para monossílabos, enquanto a média de inteligibilidade para sentenças foi de $50,3 \%$. As medidas quantitativas de inteligibilidade são clinicamente importantes porque podem monitorar mudanças durante o tratamento e documentar o nível funcional ou a adequação da comunicação.

Diversos pesquisadores avaliaram a inteligibilidade de indivíduos disártricos por meio da emissão de monossílabos e vários polissílabos. Eles identificaram que os erros produzidos por esses indivíduos foram caracterizados por omissões, substituições, distorções, acréscimos, repetições e prolongamen$\operatorname{tos}^{(7,27)}$. Esses dados concordam com os resultados do presente estudo, já que os pacientes apresentaram esses tipos de erros, principalmente na emissão de monossílabos.

\section{Prosódia}

A prosódia assume uma importância crucial na avaliação perceptiva da disartria, já que ela representa uma interação complexa de vários processos de produção de fala ${ }^{(28)}$.

Pesquisas revelam que são muito comuns as anormalidades prosódicas associadas à disartria. Geralmente, esses indivíduos apresentam proporção maior de pausas entre as palavras e no meio das palavras ${ }^{(29-30)}$. Além disso, apresentam pausas impróprias frequentes para respiração e inspiração longa e variável ${ }^{(28)}$. Essas referências corroboram com a atual pesquisa, na qual os pacientes apresentaram, em sua maioria, alteração discreta de prosódia (Tabela 5), que foi caracterizada por inadequação esporádica na marcação da sílaba tônica ou inadequação esporádica na terminação da frase ou, ainda, pausas inadequadas para a respiração.

Com relação à velocidade de fala desses indivíduos, observou-se que os pacientes apresentaram uma média de 90 palavras por minuto durante a conversação ou leitura de um texto. Estudos recentes relatam que esses indivíduos apresentam em média 85 palavras por minuto nas tarefas de leitura e uma média de 83 palavras por minuto durante a fala espontânea ${ }^{(30)}$, o que se assemelha aos achados presentes.

\section{CONCLUSÕES}

Trata-se de um estudo inicial que visou verificar o perfil populacional dos pacientes disártricos atendidos em um hospital terciário. O cenário deste estudo foi um Hospital escola, situado na cidade de São Paulo, que atende a uma grande demanda populacional. Portanto, apesar do número de casos analisados ser pequeno, os dados obtidos merecem algumas considerações. As etiologias mais comuns em nosso meio para este tipo de distúrbio de fala seguiram as observadas no mundo todo, tanto em países em desenvolvimento quanto em países desenvolvidos: o AVC e o TCE- este último, sobretudo por acidentes automobilísticos. Os tipos de disartria mais observados em nossa casuística foram a Disartria do Neurônio Motor Unilateral, muito comum no AVC, e a Disartria Flácida, comumente encontrada em pacientes pós -TCE e nos casos de AVC de Tronco Encefálico. As manifestações mais encontradas na fala destes indivíduos foram previstas pela literatura, o que reforça a idéia de que o diagnóstico clínico da disartria pode ser feito com grande acurácia, em qualquer cenário de avaliação, uma vez que não necessita de equipamentos sofisticados, mas requer uma boa análise das cinco bases motoras da fala.

Este estudo sugere que grande parte dos casos acometidos por este distúrbio poderiam ter sido evitados por meio de campanhas de educação para o trânsito e de prevenção dos fatores de risco para o AVC.

\section{AGRADECIMENTOS}

Ao Conselho Nacional de Desenvolvimento Científico e Tecnológico $(\mathrm{CNPq})$ pela concessão de fomento à pesquisa realizada. 


\begin{abstract}
Purpose: To characterize the populational profile of dysarthric patients assisted in a tertiary hospital, and to present the most frequent speech disorders found in this population. Methods: A specific protocol proposed for dysarthric patients was applied, gathering data through tasks that evaluate breathing, phonation, resonance, articulation, and prosody. Sixty protocols applied to patients evaluated at the Communication Disorders Ambulatory of the institution were the study took place were randomly selected for analysis. Data was descriptively analyzed. Results: Regarding the populational profile of the dysarthric patients attended at a tertiary hospital, it was observed prevalence of male subjects, mostly aged from 20 to 50 years. The most common types of dysarthria were flaccid and unilateral upper motor neuron. Stroke was the most prevalent etiology for this speech disorder. Regarding the motor bases evaluated, it was observed a predominance of mixed breathing, hoarse voice, and normal velar movement, although mild hypernasality was identified. Articulation was mostly severely altered, and mild prosody alterations were predominant in the investigated population. Conclusion: It was possible to draw a profile of dysarthric patients assisted in a tertiary hospital in Brazil. The most common characteristics were: short breathing cycles, hoarse hypernasal phonation, moderate to severe articulation disorders, mild prosody impairment and low speech rate.
\end{abstract}

Keywords: Dysarthria; Voice disorders; Speech acoustics; Speech; Voice quality

\section{REFERÊNCIAS}

1. Darley FL, Aronson AE, Brown JR. Differential diagnostic patterns of dysarthria. J Speech Hear Res. 1969;12(2):246-69.

2. Sellars C, Hughes T, Langhorne P. Speech and language therapy for dysarthria due to non-progressive brain damage. Cochrane Database Syst Rev. 2005;20(3):CD002088. Review.

3. Urban PP, Rolke R, Wicht S, Keilmann A, Stoeter P, Hopf HC, Dieterich M. Left-hemispheric dominance for articulation: a prospective study on acute ischaemic dysarthria at different localizations. Brain. 2006;129(Pt 3):767-77.

4. Ortiz KZ. Distúrbios neurológicos adquiridos: fala e deglutição. Barueri: Manole; 2006.

5. Wang YT, Kent RD, Duffy JR, Thomas JE, Weismer G. Alternating motion rate as an index of speech motor disorder in traumatic brain injury. Clin Linguist Phon. 2004;18(1):57-84.

6. Carrillo L, Ortiz KZ. Análise vocal (auditiva e acústica) nas disartrias. Pró-Fono. 2007;19(4):381-6.

7. Barreto SS, Ortiz KZ. Medidas de inteligibilidade nos distúrbios da fala: revisão crítica da literatura: [revisão]. Pró-Fono. 2008; 20(3):201-6.

8. Tjaden K, Wilding GE. Rate and loudness manipulations in dysarthria: acoustic and perceptual findings. J Speech Lang Hear Res. 2004;47(4):766-83.

9. Kent RD, Duffy JR, Slama A, Kent JF, Clift A. Clinicoanatomic studies in dysarthria: review, critique, and directions for research. J Speech Lang Hear Res. 2001;44(3):535-51.

10. Lotufo PA, Bensenor IM. Stroke mortality in São Paulo (1997-2003): a description using the Tenth Revision of the International Classification of Diseases. Arq Neuropsquiatr. 2004;62(4):1008-11.

11. Ferris A, Robertson RM, Fabunmi R, Mosca L; American Heart Association; American Stroke Association. American Heart Association and American Stroke Association national survey of stroke risk awareness among women. Circulation. 2005;111(10):1321-6.

12. Centers for Disease Control and Prevention (CDC). Prevalence of stroke--United States, 2005. MMWR Morb Mortal Wkly Rep. 2007;56(19):469-74.

13. Dulli D, Samaniego EA. Inpatient and community ischemic strokes in a university hospital. Neuroepidemiology. 2007;28(2):86-92.

14. Löfmark U, Hammarström A. Evidence for age-dependent educationrelated differences in men and women with first-ever stroke. Results from a community-based incidence study in northern Sweden. Neuroepidemiology. 2007;28(3):135-41.

15. Frost L, Vukelic Andersen L, Godtfredsen J, Mortensen LS. Age and risk of stroke in atrial fibrillation: evidence for guidelines? Neuroepidemiology. 2007;28(2):109-15.
16. Murdoch BE. Disartria: uma abordagem fisiológica para avaliação e tratamento. São Paulo: Lovise; 2005.

17. Isshiki N, Okamura H, Morimoto M. Maximum phonation time and air flow rate during phonation: simple clinical tests for vocal function. Ann Otol Rhinol Laryngol. 1967;76(5):998-1007.

18. Kent RD, Kent JF, Rosenbek JC. Maximum performance tests of speech production. J Speech Hear Disord. 1987;52(4):367-87. Review.

19. Inagi K, Khidr AA, Ford CN, Bless DM, Heisey DM. Correlation between vocal functions and glottal measurements in patients with unilateral vocal fold paralysis. Laryngoscope. 1997;107(6):782-91.

20. Steffen LM, Moschetti MB, Steffen N, Hanayama EM. Paralisia unilateral de prega vocal: associação e correlação entre tempos máximos de fonação, posição e ângulo de afastamento. Rev Bras Otorrinolaringol. 2004;70(4):450-5.

21. Eckel FC, Boone DR. The S/Z ratio as an indicator of laryngeal pathology. J Speech Hear Disord. 1981;46(2):147-9.

22. Dejonckere PH, Ohbens C, de Moor GM, Wieneke GH. Perceptual evaluation of dysphonia: reliability and relevance. Folia Phoniatr (Basel). 1993;45(2):76-83.

23. Lee AS, Ciocca V, Whitehill TL. Acoustic correlates of hypernasality. Clin Linguist Phon. 2003;17(4-5):259-64.

24. Theodoros D, Murdoch BE, Stokes PD, Chenery HJ. Hypernasality in dysarthric speakers following severe closed head injury: a perceptual and instrumental analysis. Brain Inj. 1993;7(1):59-69.

25. Williams RG, Eccles R, Hutchings H. The relationship between nasalance and nasal resistance to airflow. Acta Otolaryngol. 1990;110(56):443-9.

26. Haapanen ML. A simple clinical method of evaluating perceived hypernasality. Folia Phoniatr (Basel). 1991;43(3):122-32. Erratum in: Folia Phoniatr (Basel) 1991;43(4):following 203.

27. Jaeger M, Hertrich I, Stattrop U, Schönle PW, Ackermann H. Speech disorders following severe traumatic brain injury: kinematic analysis of syllable repetitions using electromagnetic articulography. Folia Phoniatr Logop. 2000;52(4):187-96.

28. Wang YT, Kent RD, Duffy JR, Thomas JE. Dysarthria associated with traumatic brain injury: speaking rate and emphatic stress. J Commun Disord. 2005;38(3):231-60.

29. Wang YT, Kent RD, Duffy JR, Thomas JE. Dysarthria in traumatic brain injury: a breath group and intonational analysis. Folia Phoniatr Logop. 2005;57(2):59-89.

30. Oliveira CR, Ortiz KZ, Vieira MM. Disartria: estudo da velocidade de fala. Pró-Fono. 2004;16(1):39-48. 